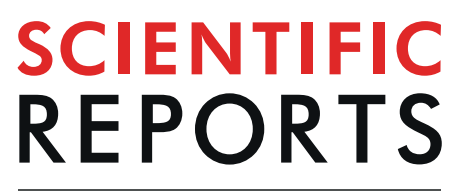

natureresearch

\title{
OPEN MicroRNA-mRNA Interactions at Low Levels of Compressive Solid Stress Implicate mir-548 in Increased Glioblastoma Cell Motility
}

\author{
Mark A. Calhoun ${ }^{1,5}$, Yixiao Cui ${ }^{1,5}$, Eileen E. Elliott ${ }^{2}$, Xiaokui Mo ${ }^{4}{ }^{4}$, Jose J. Otero ${ }^{3}$ \& \\ Jessica O. Winter (ii) ${ }^{1,2^{*}}$
}

Glioblastoma (GBM) is an astrocytic brain tumor with median survival times of $<15$ months, primarily as a result of high infiltrative potential and development of resistance to therapy (i.e., surgical resection, chemoradiotherapy). A prominent feature of the GBM microenvironment is compressive solid stress (CSS) caused by uninhibited tumor growth within the confined skull. Here, we utilized a mechanical compression model to apply CSS $(<115 \mathrm{~Pa}$ ) to well-characterized LN229 and U251 GBM cell lines and measured their motility, morphology, and transcriptomic response. Whereas both cell lines displayed a peak in migration at $23 \mathrm{~Pa}$, cells displayed differential response to CSS with either minimal (i.e., U251) or large changes in motility (i.e., LN229). Increased migration of LN229 cells was also correlated to increased cell elongation. These changes were tied to epigenetic signaling associated with increased migration and decreases in proliferation predicted via Ingenuity ${ }^{\circledR}$ Pathway Analysis (IPA), characteristics associated with tumor aggressiveness. miRNA-mRNA interaction analysis revealed strong influence of the miR548 family (i.e., mir-548aj, mir-548az, mir-548t) on differential signaling induced by CSS, suggesting potential targets for pharmaceutical intervention that may improve patient outcomes.

Glioblastoma (GBM) is a highly lethal brain tumor characterized by diffuse margins and with median survival times of 20 months from diagnosis ${ }^{1}$. The diffuse invasion of GBM cells into the brain parenchyma renders curative surgical resection and radiotherapy improbable ${ }^{2}$. Further complicating treatment, migratory cells are less proliferative $^{3}$ allowing them to evade drugs that target proliferating cells, i.e. temozolomide (TMZ), which is the first line of defense in GBM. Thus, recurrence is almost inevitable, generally occurring $\sim 9$ months post-resection ${ }^{4}$. In up to $90 \%$ of cases, recurrence occurs within a $2 \mathrm{~cm}$ margin of the tumor cavity ${ }^{5}$, implicating highly motile GBM cells that have invaded the surrounding brain parenchyma. These cells are most commonly found occupying perivascular and perineural spaces ${ }^{6}$. The perivascular niche presents unique challenges, as it co-opts host vasculature to alter the brain microenvironment ${ }^{7}$. These microenvironmental changes, among others, are part of the reason GBM has a high rate of mortality.

An important component of the GBM tumor microenvironment includes physical forces caused directly or indirectly by tumor progression. Tumor co-option of vasculature leads to leaky blood vessels ${ }^{8}$, blood stasis ${ }^{9}$, and elevated interstitial fluid pressure in the tumour ${ }^{10}$. In addition, tumor growth within the steric hindrance of the skull causes mechanical stresses within brain tissue, including compressive solid stress (CSS) in the radial direction and tension in the tangential direction. This suite of physical stimuli has implications in tumor pathophysi$o \log y^{11}$. Whereas tangential forces are most likely stored in large elements, such as axons and white matter tracts,

\footnotetext{
${ }^{1}$ Department of Biomedical Engineering, The Ohio State University, Columbus, OH, USA. ${ }^{2}$ William G. Lowrie Department of Chemical and Biomolecular Engineering, The Ohio State University, Columbus, OH, USA. ${ }^{3}$ Department of Pathology, The Ohio State University, Columbus, OH, USA. ${ }^{4}$ Center for Biostatistics and Bioinformatics, The Ohio State University, Columbus, OH, USA. ${ }^{5}$ These authors contributed equally: Mark A. Calhoun andYixiao Cui. *email: winter.63@osu.edu
} 
CSS most likely acts on normal and cancerous cells. Noticeably, CSS has been shown to compress and displace vasculature surrounding the GBM tumour ${ }^{12}$. In breast cancer cell models, CSS enhances elongation and migration $^{13,14}$, decreases proliferation, and increases apoptosis ${ }^{15,16}$. There are comparatively fewer studies in GBM, but mechanical compression has been shown to increase expression of VEGF, collagen IV and VI, and the collagen crosslinker lysyl oxidase ${ }^{17}$. Thus, CSS in GBM may be correlated to angiogenesis and vascular co-option; however, these results were based on high levels of CSS (physiological maximum of $\sim 0.21 \mathrm{kPa}$ in a mouse model ${ }^{18} \mathrm{vs} .1 \mathrm{kPa}$ in that study). Increased understanding of GBM mechanobiology, specifically as it relates to CSS, is needed to elucidate how physiologically-relevant mechanical cues are translated to GBM phenotypes relating to progression (e.g., migratory, proliferatory, and with differential genetic regulation).

CSS in GBM is associated with the midline shift, a radiological hallmark in which the tumor-affected hemisphere bulges into the contralateral hemisphere. Maximum CSS occurs at the GBM tumor boundary and decreases radially in magnitude throughout the parenchyma ${ }^{18}$. The $2-3 \mathrm{~cm}$ region beyond the tumor periphery presents a critical tumor microenvironment niche in GBM, with physical stimuli acting on invasive tumor cells that escape conventional therapy (i.e., surgical resection, chemoradiotherapy). CSS decay rapidly in a radial fashion with distance from tumour (i.e., from $\sim 100 \mathrm{~Pa}$ to $10 \mathrm{~Pa}$ in $100 \mu \mathrm{m}^{12}$ ), and CSS in infiltrative tumour decays more rapidly than that in nodular tumours ${ }^{12}$. Studies using low CSS, closer to physiological values, would better recapitulate the mechanical environment in this critical $2 \mathrm{~cm}$ radius of recurrence. Such an approach could lead to novel chemotherapeutic targets that intercept mechanical cues from the GBM microenvironment. At present, it is not well understood how GBM cell signal transduction pathways are altered in response to physical stimuli. Understanding differential regulation in the context of physical stimuli is a first step to potential pharmacological intervention and by extension, better patient outcomes.

Here, we sought to elucidate the effects of CSS on the cellular and molecular level on isolated GBM cells, focusing on the microenvironment niche within $2 \mathrm{~cm}$ of the tumor periphery. Thus, low levels of CSS (i.e., $<0.210$ $\mathrm{kPa})$ were employed. In vivo, GBM cells migrate either collectively, guided by high Racl and Cdc42 activity "leader" cells ${ }^{6}$, or as single invasive cells reciprocally interacting with host vasculature ${ }^{19}$. To mimic the collective cell migration scenario in the perivascular and perineural spaces, we employed a wound healing assay combined with an established CSS model that applies stress in $1 \mathrm{D}^{13}$, similar to radial compression forces experienced by GBM cells. This model was previously employed to study collective cell migration and cytoskeletal dynamics, and we extended this model to study both collective and single cell motility paradigms in $\mathrm{GBM}^{20}$. Whereas no in vitro model can fully mimic physiological conditions, this model facilitates access to fresh media and oxygen, decoupling CSS from other co-morbid cues in the tumor microenvironment, such as elevated interstitial fluid pressure, vascular compression, and hypoxia. In this model, we investigated migration of LN229 and U251 cells, established GBM cell lines with defined properties that permit examination of concordance with the literature. We also investigated the role of differential epigenetic signaling and predicted pathway activation using a microarray and subsequent miRNA-mRNA interaction analysis. These results suggest potential methods to mine pharmacological targets from differential signaling induced by tumor-initiated physical forces.

\section{Results}

Migration speed was enhanced by low CSS but decreased by high CSS. Tumor cells migrating at the tumor periphery and into the brain parenchyma persist after surgery and chemoradiation, presumably leading to recurrence. Thus, we constrained our experiments to levels of CSS reflective of the $2 \mathrm{~cm}$ radius of recurrence, with forces applied in $1 \mathrm{D}$, similar to radial compression forces experienced by GBM cells. CSS peaks at the tumor periphery and decreases throughout this region ${ }^{18}$. In a mouse model, CSS was measured to a maximum of $210 \mathrm{~Pa}^{18}$, so we constrained our range of interest from 0 to $115 \mathrm{~Pa}$ (i.e., roughly half of the maximum). Pressure was applied using a modified version of a model previously used to study the leader cell migration phenotype in breast tumor cells, for which physiologically relevant CSS is much higher (i.e., $\sim 800 \mathrm{~Pa})^{13}$. In this model, cells were grown on a Transwell@ insert, which facilitated access to media and prevented hypoxia. We modified this model by including a variable weight stack (Supplementary Fig. 1A) and tested the effect of CSS on GBM migration compared to controls in a wound healing assay with a gap of $500 \mu \mathrm{m}$ over a period of $18 \mathrm{hr}$ (Supplementary Fig. 1B,C).

The no pressure (i.e., no CSS, no agar cushion) and agar (i.e., no CSS) controls did not demonstrate a statistically significant difference in wound closure in LN229, but did have a statistical difference for U251 cell lines (Fig. 1), indicating that the agar cushion alone could influence migration in a detectable manner. LN229 cells migrated faster than U251 cells, as control LN229 cells closed $57.0 \pm 3.3 \%$ of the gap, whereas control U251 cells closed only $36.7 \pm 3.0 \%$ of the gap. For LN229 cells at $23 \mathrm{~Pa}$, the maximum migration rate observed, wound closure was significantly faster than the control, with $23.2 \pm 4.3 \%$ more gap closure over $18 \mathrm{hr}$, equivalent to a $\sim 1.4 \mathrm{x}$ increase $(\mathrm{p}=0.0062)$. U251 cells also had a statistically significant peak in wound closure at $23 \mathrm{~Pa}$, closing $17.8 \pm 4.6 \%$ more of the gap than the control $(\mathrm{p}=0.0006)$, a $\sim 1.5 \mathrm{x}$ increase. At the highest CSS investigated of $115 \mathrm{~Pa}, \mathrm{LN} 229$ cells exhibited negative wound closure compared to the control, whereas U251 cells closed $13.6 \pm 5.3 \%$ more of the gap than the control $(\mathrm{p}=0.0017)$. Thus, $\mathrm{U} 251$ cells had a positive differential wound closure at all levels of CSS. This data extends previous findings of increased cell migration under CSS to GBM cancers. Additionally, it demonstrates two migratory responses to CSS: a dramatic response in LN229 cells and a minimal response in U251 cells.

Two cell morphology populations were observed. Next, we investigated the influence of CSS on cell morphology, which is closely related to many cell processes, including adhesion, contractility, and migration ${ }^{21}$. To recapitulate GBM migration through the brain parenchyma, cells were grown in a non-confluent monolayer that permitted observation of single cell morphology (Fig. 2). Qualitatively, a mixed population of rounded (Fig. 2A) and elongated cells (Fig. 2B) were present across all conditions, though their proportions varied. To 


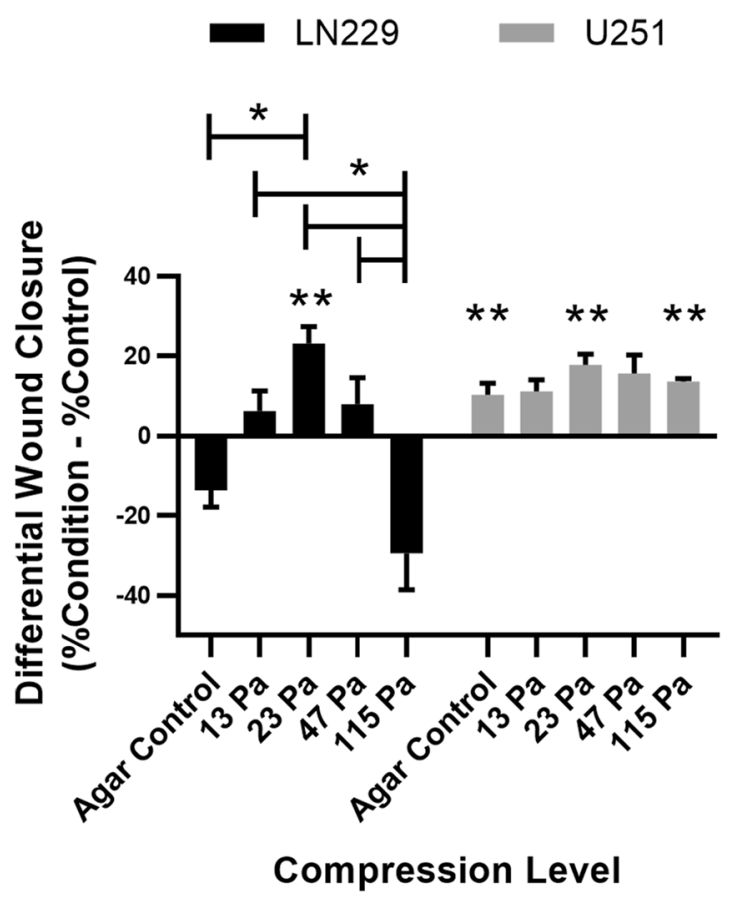

Figure 1. Collective cell migration reaches a maximum at $23 \mathrm{~Pa}$ CSS in LN229 and U251 cells. Differential wound closure: the difference of each compression level (agar control, $13 \mathrm{~Pa}, 23 \mathrm{~Pa}, 47 \mathrm{~Pa}$, and $115 \mathrm{~Pa}$ ) from its corresponding experimental control. Levels connected by a star $(*)$ are statistically significant at $\alpha=0.05$. Conditions marked with two stars $(* *)$ are statistically significant compared to their control for each cell type at $\alpha=0.01$ after Bonferroni correction.

quantify morphology, aspect ratio (AR), the ratio of length to width for which $A R=1$ is a perfect circle and higher values $(\mathrm{AR} \geq 2)$ indicate more elongated cells, was employed. Corresponding cell area was also assessed (Supplementary Fig. 2). For LN229 cells, the percent elongated cells was significantly higher at $23 \mathrm{~Pa}$ compared to the agar control and the highest CSS employed of $115 \mathrm{~Pa}(\mathrm{p}=0.0243,0.0007$, respectively) (Fig. 2C). At $23 \mathrm{~Pa}$, $39.1 \pm 4.4 \%$ of cells were elongated compared to only $21.0 \pm 3.9 \%$, or roughly half that amount, in the agar control. Conversely, U251 cells maintained a relatively high percent elongated cells across pressures $<115 \mathrm{~Pa}$. On average, U251 cells were $33.0 \pm 3.0 \%$ elongated, whereas LN229 cells were $23.8 \pm 2.2 \%$ elongated. Projected cell area results (Supplementary Fig. 2) were inversely proportional to those of AR, consistent with the assumption of incompressibility (i.e., constant volume). Cells generally had lower cell area at intermediate conditions, when AR was higher, and the largest cell areas at the $115 \mathrm{~Pa}$ when most cells were rounded. Morphology results mirrored those of migration, although U251 cells had significantly lower percent elongated cells at $115 \mathrm{~Pa}(5.8 \pm 1.9 \%$, $\mathrm{p}=0.0343$ ), which was more pronounced than declines in migration.

GBM cell elongation was further analyzed using AR histograms for each CSS level that were fit with 1- or 2-term probability density functions (PDFs) to statistically test for the presence of a second population (Fig. 2D,E). The fit for each lognormal curve was excellent $\left(\mathrm{R}^{2} \geq 0.91\right)$. LN229 cells displayed a second cell population with higher AR in all conditions except $115 \mathrm{~Pa}$ (Fig. 2D). LN229 control and agar samples had relatively small second AR populations with peaks at 2.12 and 2.19, respectively (Table 1). At 23 and $47 \mathrm{~Pa}$, these peaks shifted to higher AR with maxima at 2.63 and 2.66, respectively. Additionally, the $23 \mathrm{~Pa}$ secondary AR peak was relatively larger than the others, at roughly the same height as its primary peak. The secondary peak of $23 \mathrm{~Pa}$ was also narrower than that of $47 \mathrm{~Pa}$ (variance $\sigma^{2}$ of 0.0001 vs 0.0015 ). (Supplementary Table 1 ). U251 cell response differed from that of LN229 cells (Fig. 2E). Only the agar control and $115 \mathrm{~Pa}$ conditions had secondary peaks (Table 1). However, neither was experimentally relevant as both were $\mathrm{AR}<2$ (i.e. not elongated). These data further illustrate GBM cell response to CSS: a dramatic response associated with an induced elongated cell subpopulation in LN229 cells and a minimal response independent of cell elongation below $115 \mathrm{~Pa}$ in U251 cells.

Differential gene expression, correlated to increased migration, is induced at low CSS in LN229 cells. The mechanism by which physiologically relevant levels of CSS transduce increased migration and elongation in GBM cells is presently unclear. To gain a comprehensive perspective of changes induced by CSS, RNA regulation of LN229 cells at $23 \mathrm{~Pa}$ in the wound healing assay was compared to that of control cells after $18 \mathrm{hr}$ using the GeneChip ${ }^{\mathrm{TM}}$ Human Transcriptome Array 2.0 microarray. Of the 135750 RNAs analyzed, there were 2727 differentially expressed RNAs at $23 \mathrm{~Pa}$ compared to the control ( $\mathrm{p}<0.05$, false discovery rate (FDR) $\mathrm{p}<0.05$, absolute fold change $>2$ ) (Supplementary Fig. 3). The three most upregulated RNA were STC1, TMEM45A, and $C A 9$ with fold changes of $12.79,9.64$, and 7.75 , respectively (Table 2 ). These and other differentially regulated RNAs of note (Table 2) are associated with poor prognosis, chemoresistance, recurrence, increased motility, and angiogenesis. Additionally, 27 precursor miRNA were differentially regulated, 11 of which correlate with 

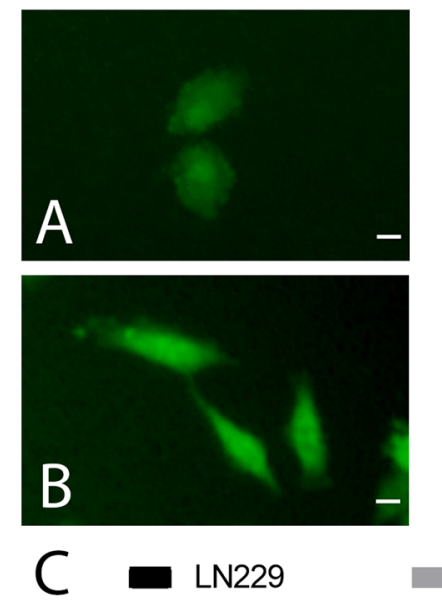

U251
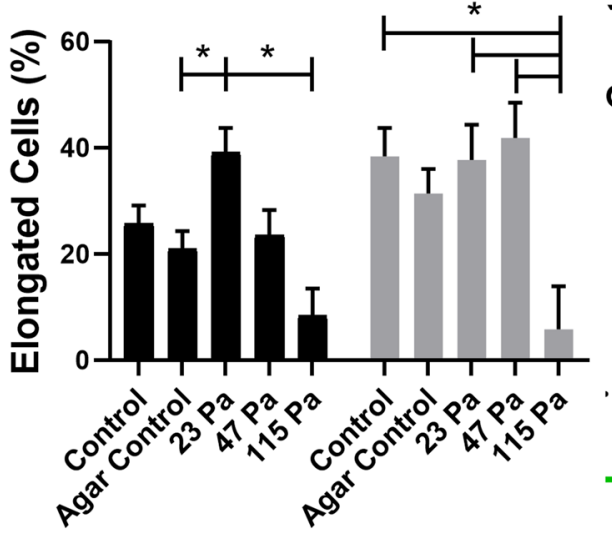

\section{Compression Level}

D

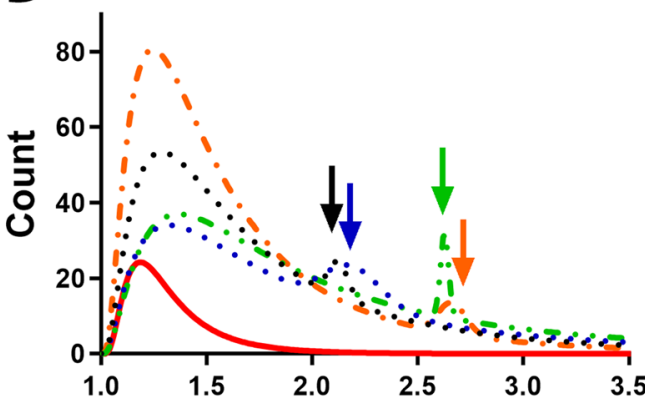

E $\quad \therefore \quad$ Aspect Ratio

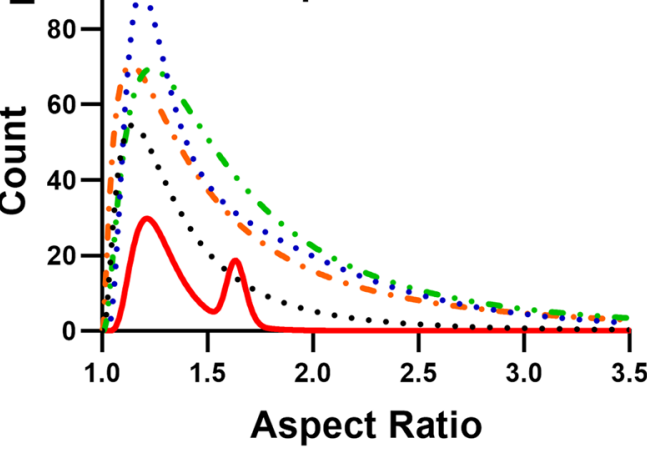

... Agar Control

- $23 \mathrm{~Pa} \quad \rightarrow 47 \mathrm{~Pa} \quad-115 \mathrm{~Pa}$

Figure 2. CSS induces an elongated subtype in single LN229 cells under CSS. Representative images of LN229 cells show rounded morphology in the agar control (A) and elongated cells at $23 \mathrm{~Pa}(\mathbf{B})$. Scale bar $=20 \mu \mathrm{m}$. (C) Percent elongated cells (AR $\geq 2$ ) for LN229 and U251 cells. Levels connected by a star (*) are statistically significant at $\alpha=0.05$. Probability density functions of LN229 (D) and U251 (E) cell aspect ratio (AR) at increasing levels of CSS. Arrows indicate the second population peaks.

\begin{tabular}{|l|l|l|l|l|}
\hline \multirow{5}{*}{ LN229 } & $\begin{array}{l}\mathbf{1}^{\circ} \text { Peak } \\
\text { Mode }\end{array}$ & $\begin{array}{l}\mathbf{2}^{\circ} \text { Peak } \\
\text { Mode }\end{array}$ & $\mathbf{R}^{2}$ \\
\hline & Control & 1.29 & 2.12 & 0.94 \\
\cline { 2 - 5 } & Agar Control & 1.32 & 2.19 & 0.98 \\
\cline { 2 - 5 } & $23 \mathrm{~Pa}$ & 1.37 & 2.63 & 0.91 \\
\cline { 2 - 5 } & $47 \mathrm{~Pa}$ & 1.24 & 2.66 & 0.99 \\
\cline { 2 - 5 } & $115 \mathrm{~Pa}$ & 1.19 & - & 0.95 \\
\hline \multirow{5}{*}{ U251 } & Control & 1.15 & - & 0.97 \\
\cline { 2 - 5 } & Agar Control & 1.18 & 1.68 & 0.97 \\
\cline { 2 - 5 } & $23 \mathrm{~Pa}$ & 1.24 & - & 0.97 \\
\cline { 2 - 5 } & $47 \mathrm{~Pa}$ & 1.14 & - & 0.98 \\
\cline { 2 - 5 } & $115 \mathrm{~Pa}$ & 1.21 & 1.63 & 0.96 \\
\hline
\end{tabular}

Table 1. Lognormal Curve Fitting Parameters of Aspect Ratio (AR) Distributions.

prognosis, treatment resistance, angiogenesis, proliferation, or invasion in human GBM (Table 3). Implication of CA9, ITGA3, and let-7i may explain increased migration and elongation results observed here ${ }^{22,23}$. However, differential expression of selected RNAs and miRNAs alone does not provide enough context to identify interactions or correlation to clinically relevant outcomes.

Pathway analysis predicts changes in cell function and HIF-1 pathway activation at low CSS. To visualize pathways dependent on differentially regulated targets, a total of 489 protein (FDR $\mathrm{P}<0.05$, FC $>2$ ) coding genes were analyzed using Ingenuity ${ }^{\circledR}$ Pathway Analysis (IPA). The analysis compared the 


\begin{tabular}{|l|l|l|l|}
\hline & $\begin{array}{l}\text { Fold } \\
\text { change }\end{array}$ & $\begin{array}{l}\text { FDR } \\
\text { p-value }\end{array}$ & Cancer-Related Effects \\
\hline STC1 & 12.79 & 0.0002 & Poor prognosis $^{55}$ \\
\hline TMEM45A & 9.64 & 0.0002 & Progression, chemoresistance $^{56}$ \\
\hline CA9 & 7.75 & 0.0011 & Increased motility $^{57}$ \\
\hline ITGA3 & 2.34 & 0.0245 & Motility $^{58}$ \\
\hline FOXN2 & -2.63 & 0.0386 & Poor prognosis $^{59}$ \\
\hline Ly6k & 4.83 & 0.0072 & Recurrence $^{60},{\text { tumor } \text { grade }^{61}}^{61}$ \\
\hline CTGF & 2.17 & 0.0314 & Angiogenesis, poor prognosis $^{62}$ \\
\hline VEGFA & 3.02 & 0.0072 & Angiogenesis $^{63}$ \\
\hline VEGFB & 2.04 & 0.0177 & Angiogenesis $^{64}$ \\
\hline
\end{tabular}

Table 2. Selected mRNA Differentially Regulated Under 23 Pa CSS.

\begin{tabular}{|c|c|c|c|c|}
\hline miRNA & $\begin{array}{l}\text { Fold } \\
\text { change }\end{array}$ & Glioma-Related Effects & miRNA & $\begin{array}{l}\text { Fold } \\
\text { change }\end{array}$ \\
\hline \multirow{2}{*}{ mir-31-HG } & \multirow{2}{*}{-2.03} & \multirow{2}{*}{ Survival $^{65}$} & mir-563 & -2.38 \\
\hline & & & mir-569 & -2.55 \\
\hline mir-100-HG & -2.63 & Radioresistance $^{66}$ & mir-604 & -3.06 \\
\hline mir-181-A1-HG* & -4.2 & Prognosis, Radioresistance ${ }^{67,68}$ & mir-4477-B & -2.21 \\
\hline$m i r-181-B 1^{*}$ & -2.9 & Prognosis, Radioresistance ${ }^{67,68}$ & mir-6125 & -2.2 \\
\hline mir-421 & -2.05 & Invasion, Radioresistance ${ }^{69}$ & mir-6839 & -2.43 \\
\hline mir-423 & -3.28 & Angiogenesis, Chemoresistance ${ }^{70}$ & mir-7978 & -2.78 \\
\hline mir-454 & -2.22 & Prognosis $^{71}$ & mir-8063 & -2.7 \\
\hline mir-548-AJ2* & -2.16 & Prognosis, survival ${ }^{32,33}$ & mir-924 & -3.32 \\
\hline $\operatorname{mir}-548-A Z^{*}$ & -2.03 & Prognosis, survival ${ }^{32,33}$ & mir-943 & 2.02 \\
\hline mir-548-T* & -2.3 & Prognosis, survival ${ }^{32,33}$ & mir-3614 & 2.02 \\
\hline \multirow[t]{5}{*}{ let-7i } & -3.35 & Invasion, Proliferation ${ }^{22,23}$ & mir-8085 & 2.06 \\
\hline & & & mir-4640 & 2.12 \\
\hline & & & mir-6884 & 2.19 \\
\hline & & & mir-3611 & 4.17 \\
\hline & & & mir-6787 & 4.95 \\
\hline
\end{tabular}

Table 3. All miRNA Differentially Regulated at $23 \mathrm{~Pa}$ CSS. *denotes role of miR family where unique isoform data is unavailable. The miRNA on the left have been previously implicated in GBM and are listed with the known effects. The miRNA on the right are unsubstantiated in the GBM literature.

differentially regulated gene targets with existing pathway networks and generated 3 major outputs: top canonical pathways, top molecular and cellular functions, and top upstream regulators. There were 45 canonical pathways ( $\mathrm{P}<0.05$, ranked by overlap of target molecules out of total molecules in the pathway) identified to be altered by low CSS at $23 \mathrm{~Pa}$. Within the top canonical pathways, IPA predicted an activated state for gluconeogenesis I $(\mathrm{z}=2.236)$, glycolysis $\mathrm{I}(\mathrm{z}=2.646)$, and UDP-N-acetyl-D-galactosamine biosynthesis II $(\mathrm{z}=2)$ as indicated by a $\mathrm{z}$ score higher than 2 (Fig. 3). Additionally, IPA predicted cellular movement, cell-to-cell signaling and interaction, and carbohydrate metabolism to be increased $(z>2)$. Interestingly, chromosomal congression of chromosomes, which is the first step in mitosis, was predicted to be decreased $(z=-2)$, suggesting possible decreases in proliferation (Fig. 3, Supplementary Table 2).

IPA software also predicted upstream regulators activated or inhibited by treatment with $23 \mathrm{~Pa}$ CSS. HIFA $(\mathrm{z}=4.85$, $\mathrm{p}$-value $=1.77 \mathrm{E}-17)$ and $\operatorname{ARNT}(\mathrm{z}=3.67$, $\mathrm{p}$-value $=2.69 \mathrm{E}-14)$, which encode the two subunits of hypoxia-inducible factor (HIF)- 1 , HIF- $1 \alpha$ and HIF- $1 \beta$, respectively, were the top two activated upstream regulators. Additionally, $E G L N(\mathrm{z}=-4.02$, p-value $=4.96 \mathrm{E}-12)$, a HIF prolyl hydroxylase, was inhibited. Together, this predictions suggests that $23 \mathrm{~Pa}$ CSS significantly activates the HIF-1 signaling pathway. HIF-1 is commonly overexpressed in human cancers and is associated with cell apoptosis, proliferation, invasion, and angiogenesis ${ }^{24,25}$. NUPR1 $(\mathrm{z}=4.356$, $\mathrm{p}$-value $=1.49 \mathrm{E}-12)$ was also predicted to be activated. NUPR1 is upregulated in response to stress and has a promoting effect on cancer cell metastasis and resistance to therapy ${ }^{26,27}$. These results hint toward a possible mechanism of cell response to low CSS values.

miRNA-RNA interaction analysis indicate miR548 family in CSS-mediated responses. Studies demonstrating the effect of a specific mRNA or miRNA on a cancer-related metric (e.g., invasion) are often performed using viral transfection to demonstrate a correlation. Whereas this is useful for understanding a single gene, it is unclear what, if any, effect will result in the context of a complex microenvironment, such as the changes induced by a mechanical stimulus here. To identify interactions between RNAs and miRNAs identified in microarray analysis and cancer-related metrics, we performed miRNA-RNA interaction analysis. Specifically, 


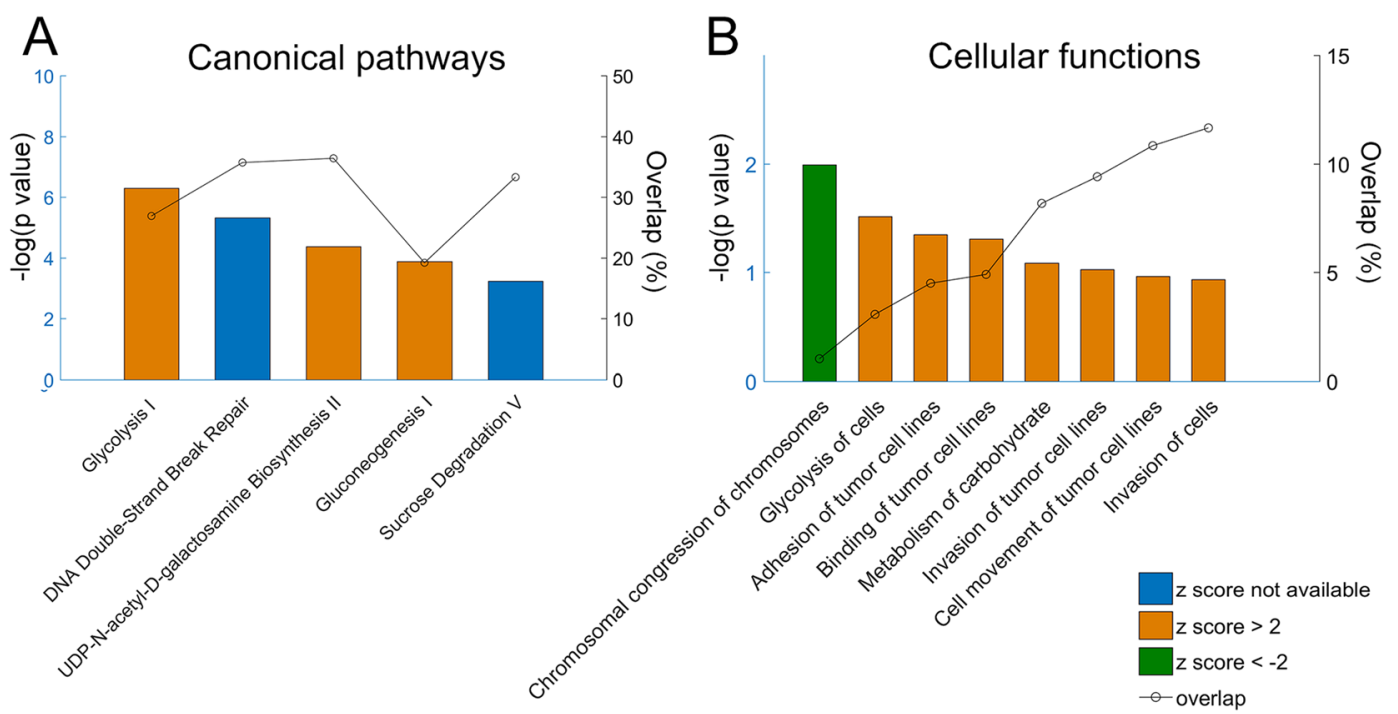

Figure 3. IPA analysis of canonical pathways and cellular functions at low CSS (23 Pa). (A) Top canonical pathways ranked by $-\log (\mathrm{p}$ value $)$. Overlap, indicated on the right axis, is calculated as the number of differentially regulated genes in the pathway over the number of total genes in that pathway. (B) Top molecular and cellular functions ranked by $-\log (p$ value). Overlap, shown on the right axis, is the number of differentially regulated genes related to that function over total number of differentially expressed genes. $Z$ scores higher than 2 indicate pathway activation/ cell function increase, $\mathrm{z}$ scores lower than -2 indicate pathway suppression/ cell function decrease.

we cross-referenced the 27 precursor miRNAs (Table 3) and their differentially regulated target mRNAs identified by microarray analysis with 517 pathways, across 4 databases, representing 7 cancer progression-related classifications, using custom Python scripts (Supplementary material) and miRPathDB ${ }^{28}$. Pathways were classified into one of seven pathway groups using inclusion/exclusion criteria to facilitate analysis (Supplementary Table 3, 4). This analysis yielded various heat maps, with nodes depicting either the number of intersections, percent overlap, or cumulative absolute fold change (FC) (Fig. 4). A miRNA was defined as having an intersection with a pathway if it had significantly more target mRNA in the pathway than expected by random chance $(\mathrm{p}<0.05)$. Percent overlap was defined as the number of differentially regulated target mRNAs normalized to the total number of target mRNAs for a given miRNA-pathway pair. Cumulative absolute FC was the sum of the absolute FC of all differentially regulated target mRNAs for a given miRNA.

The Motility pathway group had the highest number of intersections, with 4 pathways intersecting more than 20 of the 27 miRNA (Fig. 4A). The 4 pathways were cell projection morphogenesis, cell projection, plasma membrane bounded cell projection, and cell projection organization. These are all related to cell projections and may help explain increased elongation seen experimentally. The DNA Repair, Apoptosis, and Miscellaneous pathway groups were not strongly targeted. Increased DNA repair and apoptosis avoidance are linked to treatment resistance, as demonstrated by the effect of MGMT methylation on patient prognosis ${ }^{29}$. MGMT encodes a DNA-repair protein that allows cell survival after treatment with alkylating agents (e.g., TMZ) ${ }^{30}$. Microarray results implicated dysregulation of several genes that could be associated with development of resistance to therapy (Tables 2, 3). However, miRNA-mRNA interactions analysis did not implicate DNA repair or apoptosis-avoidance mechanisms, at least over the time points evaluated in this study. Whereas several of these processes can be rapid in individual cells (e.g., minutes to hours), the methods employed here are not sensitive to population-level genetic shifts that may take longer to manifest.

Percent overlap (Fig. 4B) and cumulative absolute FC (Fig. 4C) were used to quantify the influence each miR exerted over a given pathway in terms of quantity and quality, respectively. The Cancer pathway group was used as a standard for comparison, as these pathways are expected to be strongly dysregulated in GBM. Cancer had the highest percent overlap (Fig. 5A), as expected, but was eclipsed by the Motility pathway group with respect to cumulative absolute FC (Fig. 5B). Percent overlap for the Motility pathway group was about half as large as the Cancer pathway group, whereas cumulative absolute FC was $\sim 2.5 \mathrm{x}$ larger. Significant dysregulation of the Motility pathway group was expected based on migration and morphology results. These results suggest that increased motility observed under CSS is related to strong differential regulation of fewer genes. The Cell Cycle pathway group also demonstrated relatively high dysregulation compared to the Cancer pathway group. Percent overlap for the Cell Cycle pathway group was around half as large as the Cancer pathway group, similar to the Motility pathway group. However, its cumulative absolute FC was similar to the Cancer pathway group (2.32, 2.69, respectively). This result corroborates IPA results, suggesting altered proliferation at $23 \mathrm{~Pa}$ CSS. Additionally, miRNAs were classified based on their relevance to GBM in peer-reviewed literature, indicating a significantly strong influence as measured by percent overlap and cumulative absolute FC (Fig. 5C,E). This result hazards caution when extending these data to other cancers, at least in the context of mechanobiology. 
A

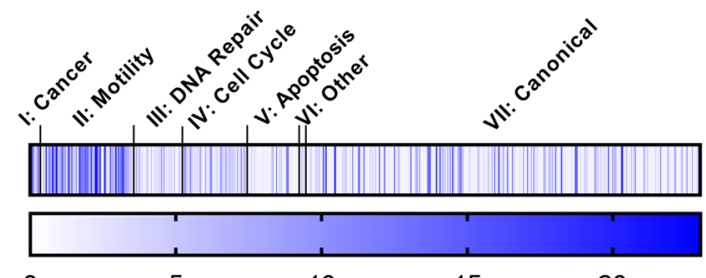

B

10

15

20
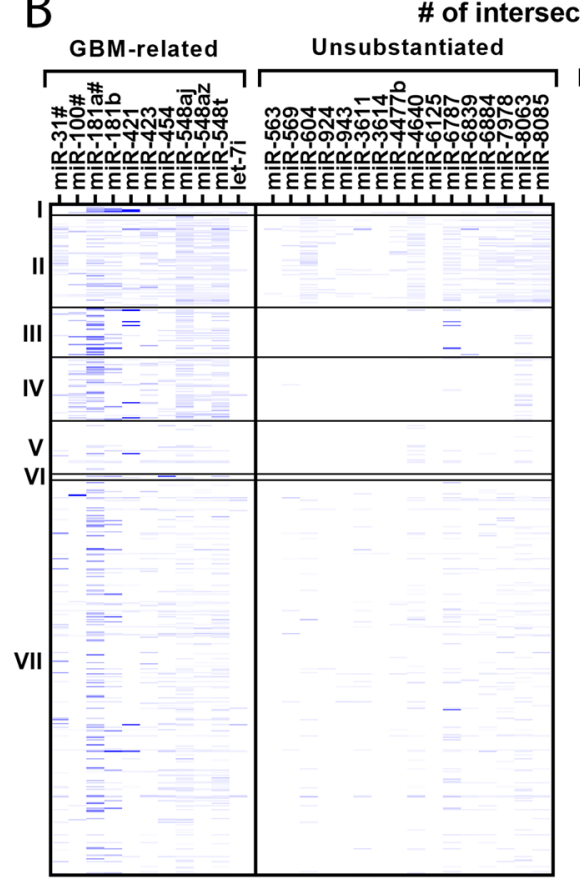

GBM-related
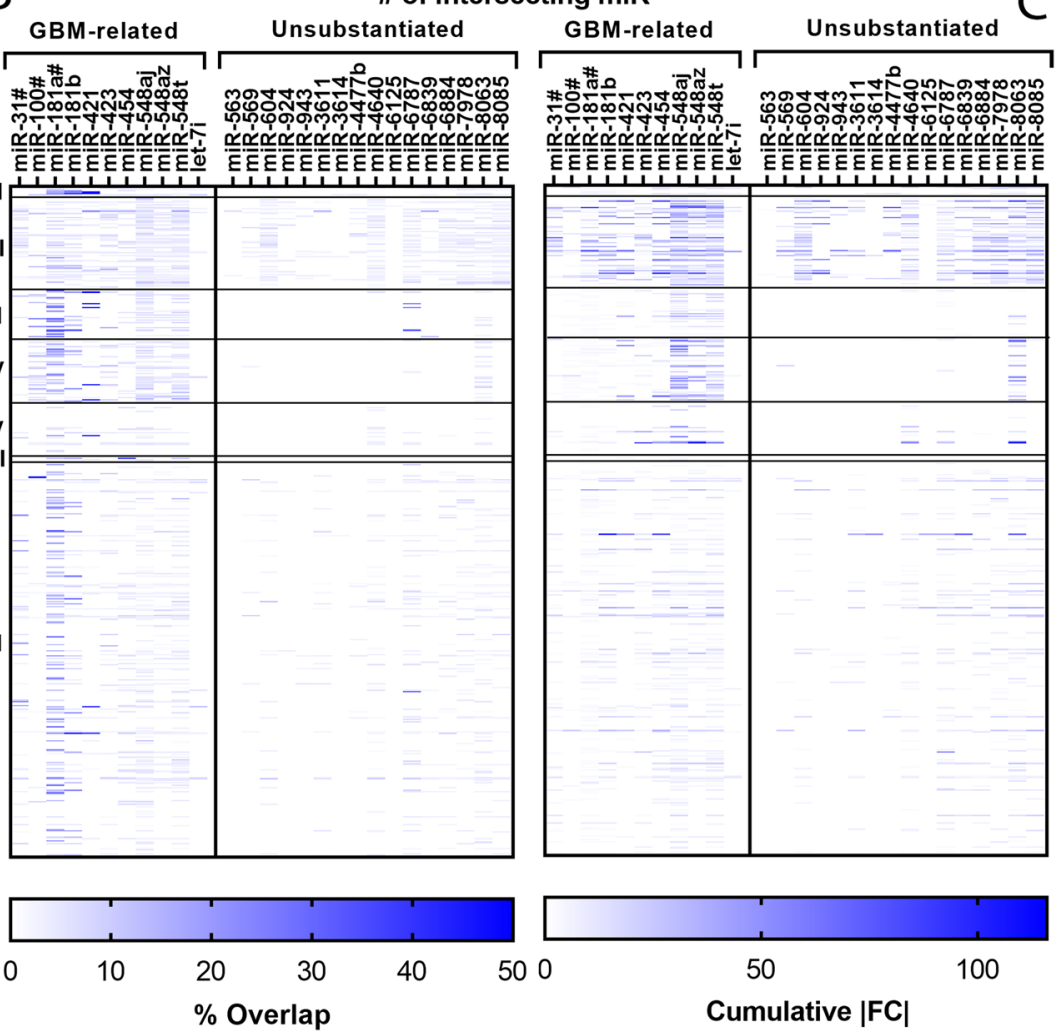

Cumulative $|\mathrm{FC}|$

Figure 4. miRNA-mRNA interactions show miRNA and pathway group dysregulation. (A) The total number of miRNA with target mRNA that interact with a given pathway. (B) Percent overlap for each miRNA-pathway pair. Percent overlap: the \% of mRNA targets that were differentially regulated. (C) Cumulative absolute fold change (FC) for each miRNA-pathway pair. Cumulative absolute FC: the cumulative unsigned fold change of the differentially regulated targets. A total of 167 pathways were classified into functional groups: I: Cancer, II: Motility, III: DNA Repair, IV: Cell Cycle, V: Apoptosis, VI: Miscellaneous, VII: Canonical. The 27 miRNA were split by their relevance to GBM. miRNA denoted with a hashtag (\#) denotes a substitution for a miRNA included in a host gene.

The maximum percent overlap was 50\% for the WikiPathways ATM Signaling Network in Development and Disease. The maximum cumulative absolute FC was 115.98 in the GO Biological Process Apoptotic Process. Although these were maxima are in the Canonical and Apoptosis pathway groups, respectively, neither pathway group as a whole was strongly affected. In grouped analysis, the Canonical and Apoptosis pathway groups demonstrated the lowest percent overlap, less than $\sim 0.55$ compared to the Cancer pathway group at 2.74. Their cumulative absolute FC was also less than roughly half of the Cancer pathway group at 2.69. These results suggest that altered apoptosis is not relevant in this model at this time point.

The influence of each miRNA was also quantified as a means of identifying potential therapeutic targets. By percent overlap, mir-181a had the strongest influence, followed by mir-548aj, mir-181b, and mir-548t (Fig. 5D). By cumulative absolute FC, mir-548aj, mir-548t, and mir-548az had the strongest influence (Fig. 5F). Downregulation of the $m i R 181$ family is associated with glioma progression ${ }^{31}$, whereas the $m i R 548$ family is associated with prognosis and survival ${ }^{32,33}$. These results suggest that both families are potential therapeutic targets. However, mir-181A1HG was detected in the microarray and mir-181a was substituted to match isoform data availability (Fig. 4). This warrants caution, as host gene regulation may not necessarily match that of the miRNA ${ }^{34}$. These results suggest that the miR548 family exerts a strong influence on pathway groups implicated in GBM response to CSS and may be the best candidate for pharmaceutical intervention. 
A
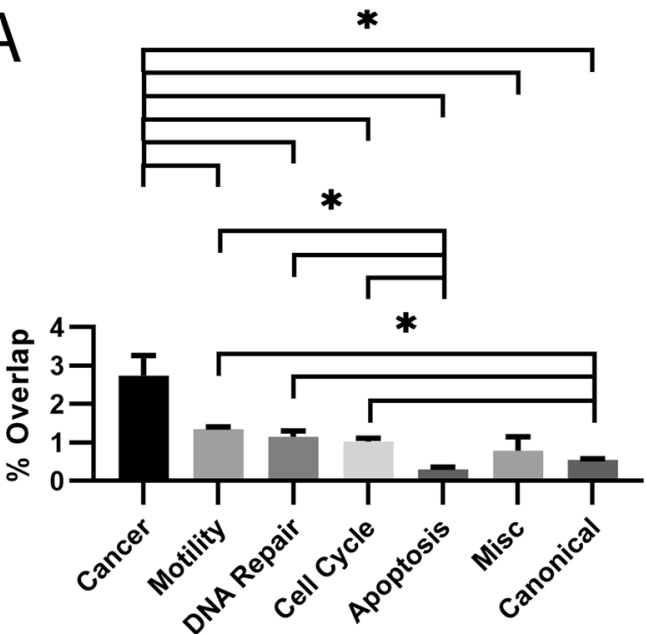

B

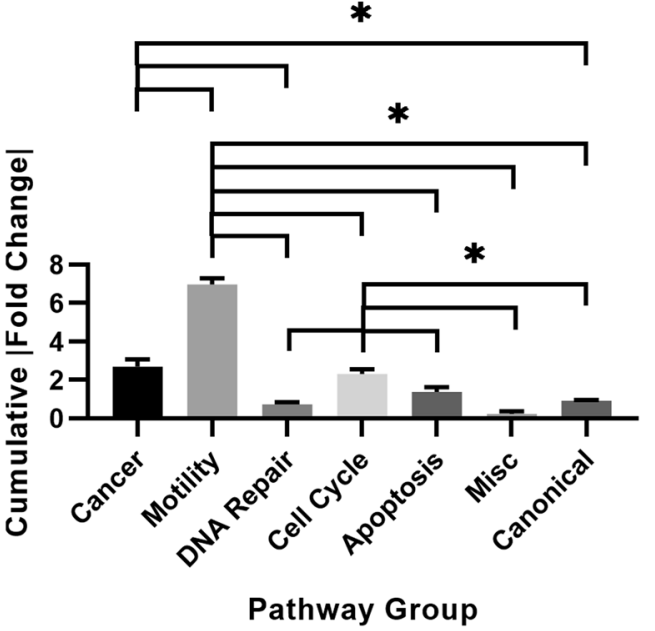

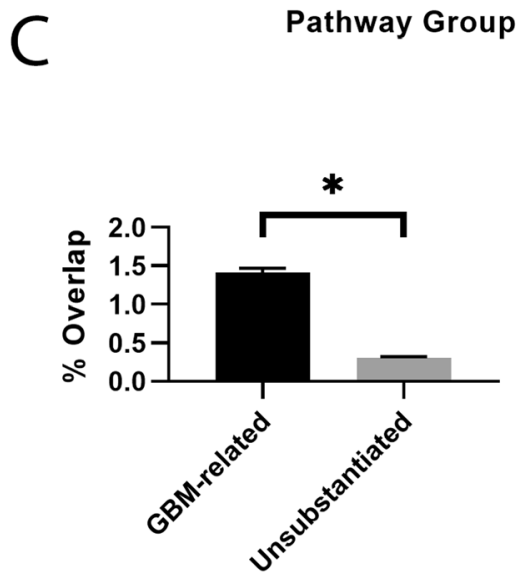

$D_{6}$

$\mathrm{E}$

miR Group

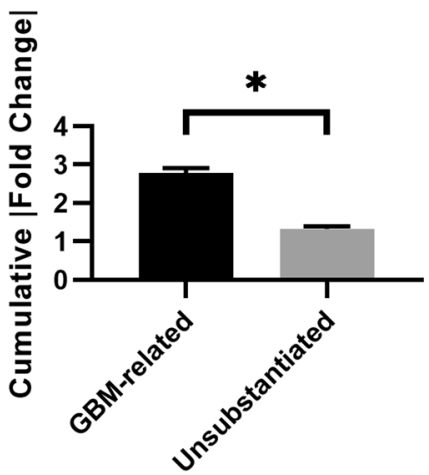

miR Group

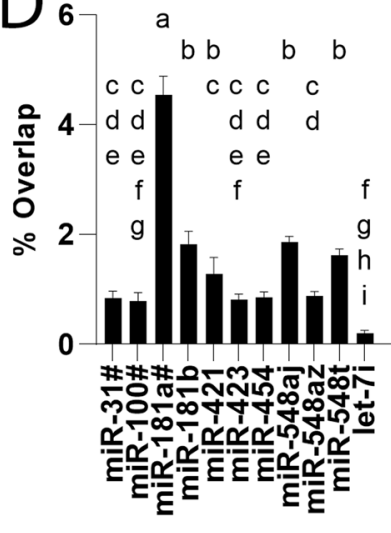

a

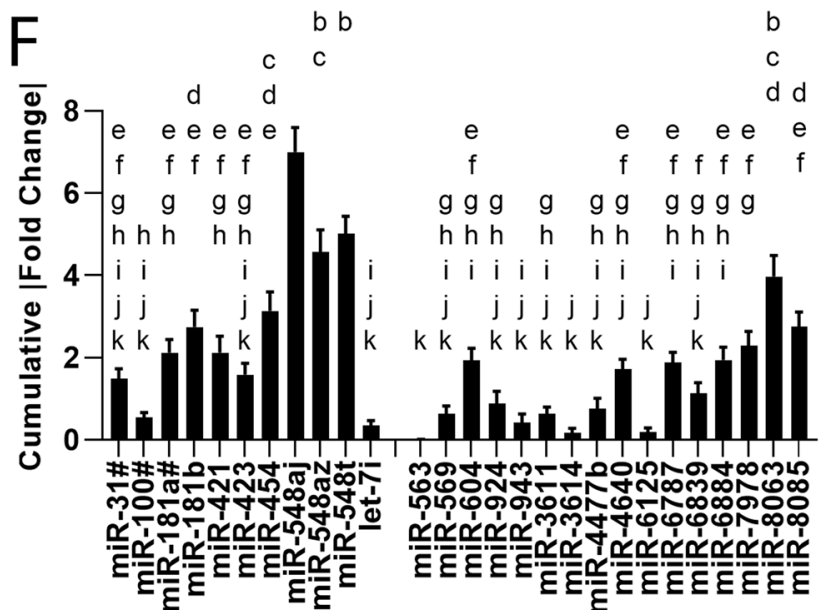

Figure 5. Canonical, Motility, and Cell Cycle Pathway groups and the miR548 family are predicted to be altered under $23 \mathrm{~Pa}$ CSS based on mRNA-miRNA interaction analysis. (A) Percent overlap and (B) cumulative absolute fold change (FC) for each pathway group. (C) Percent overlap and (D) cumulative absolute FC for each miR group based on literature relevance to GBM. Levels connected by a star $(*)$ are statistically significant at $\alpha=0.05$. (E) Percent overlap and (F) cumulative absolute FC for each miRNA across all pathways. Letters not connected by the same letter are statistically different at $\alpha=0.05$. miRNA denoted with a hashtag (\#) denotes a substitution for a miRNA included in a host gene.

\section{Discussion}

This study presents initial work toward identification of pathways involved in GBM response to CSS, particularly at levels (i.e., $<115 \mathrm{~Pa}$, or roughly half of maximum observed in a mouse model ${ }^{18}$ ) that might be observed in the $2 \mathrm{~cm}$ region of recurrence adjacent to the tumor periphery. This study also examined the relationship 
between tumor-induced physical force and aggressive tumor features to identify possible positive feedback loops. Specifically, cell migration, morphology, and differential gene signaling were examined for well-characterized LN229 and U251 GBM cell lines. Both LN229 and U251 cell lines exhibited maximal migration at applied CSS of $23 \mathrm{~Pa}$, compared to little/no statistical difference observed for lower pressures indicative of a "normal" brain environment (Fig. 1). According to the best available approximation of CSS distribution through the brain ${ }^{12}$, GBM cells experience this level of CSS at 5-10\% of the tumor radius away from the tumor. As such, it is possible that CSS at the tumor periphery temporarily "boosts" GBM cell migration. These results are consistent with previous findings. GBM cells display high sensitivity to their mechanical microenvironment. For example, we have shown that U87 GBM cells grown on $50 \mu \mathrm{m}$ thick electrospun fiber mats ${ }^{35}$ or patient-derived GBM cells cultured in $<25 \mu \mathrm{m}$ thick hydrogels ${ }^{36}$ are sensitive to the mechanics of their underlying supports. GBM cells also increase migration in response to low linear flow rates $(<1 \mu \mathrm{m} / \mathrm{s})^{37}$.

Increased migration at $23 \mathrm{~Pa}$ likely results from cross-talk between mechanically-responsive integrins and metabolic pathways. Microtubules resist and dissipate the CSS mechanical load, transmitting force to the extracellular environment via focal adhesions ${ }^{38}$. At focal adhesions, mechanical forces converge with biochemical signaling, initiating signal transduction ${ }^{38}$. There is significant cross-regulation between integrins and metabolic pathways (i.e., $A M P K, m T O R, H I F-1)^{39}$. In particular, integrins contribute to and are necessary for differential signaling in cancer cells that leads to increased glycolysis and biosynthetic precursor production ${ }^{39,40}$. We detected a significant upregulation of ITGA3 $(\mathrm{FC}=2.34$, FDR $\mathrm{P}=0.0245)$, encoding integrin $\alpha_{3}$, in cells treated with CSS. Upregulation of integrin $\alpha_{3}$ has been detected in cells treated with elevated CSS ${ }^{14}$ and hydrostatic pressure ${ }^{41}$. The downstream signaling of integrin $\alpha_{3}$ is illustrated in the focal adhesion network, as generated from WikiPathways (Supplementary Fig. 4).

We also observed differences in migratory behaviors between the two cell lines. For LN229 cells, wound closure decreased below that of the control at high levels of CSS, whereas for U251 cells wound closure was equivalent to or higher than the control at all CSS values investigated. These differences in migratory response were also correlated to morphology. We observed a mechanically-induced, elongated subtype in LN229 cells, present at all pressures except the highest employed, but that was most pronounced at $23 \mathrm{~Pa}$ CSS (Fig. 2). This subtype was not observed in U251 cells (Table 1). This behavior may be similar to the collective migration leader cell phenotype observed in breast cancer cell compression studies, which is induced by forced filopodia extrusion into the cell-free area on one side of the cell ${ }^{13}$. However, this is unlikely. Our study employed non-confluent, monolayer culture in which cell-free area was available on all sides. Additionally, we neither observed nor measured leader cell formation in wound healing assays. Previous studies also used much higher levels of CSS (i.e., $773 \mathrm{~Pa}$ vs $0-115 \mathrm{~Pa}$ here), which was physiologically relevant to much stiffer breast cancer tissue. As such, the degree of forced extrusion into the cell-free area would be proportionally lower. The elongated morphologies observed here may be adopted simply as a result of their energetic favorability; an elongated cell has a lower projected cell area, minimizing CSS versus a round cell. However, LN229 cells did not uniformly elongate, instead forming a secondary peak with specific AR maximum of 2.6 (Table 1). This behavior suggests a phenotypic change. More experiments are needed to understand differences in single cell and collective cell responses to CSS in LN229 cells. Further, it is important to note that this study examined isolated GBM cell response, whereas the tumor microenvironment is a complex niche with many interconnected cues. Thus, the collective tumor response may differ from that of isolated GBM cells and can best be discerned by increasing model complexity with the addition of stromal or immune cells or through in vivo studies. Such studies would elucidate differences between isolated GBM response and effects of cell-cell signaling. In vivo studies would be particularly informative as CSS is likely distributed through many structural elements aside from GBM cells, such as blood vessels, extracellular matrix, and stromal cells.

Physiologically, GBM cells elongate by sending out long projections along white matter tracts and the perivascular space ${ }^{42}$. Thus, the observed correlation of elongation and increases in migration for LN229 cells (Figs. 1, 2) may result from activation of shared pathways. To identify potential pathways involved in GBM CSS response, we assessed LN229 cell signaling at $23 \mathrm{~Pa}$ versus controls with no applied CSS using a Human Transcriptome Array. Changes in several mRNA and precursor miRNA associated with tumor aggressiveness were observed (Tables 2, 3). Low CSS level at $23 \mathrm{~Pa}$ was predicted to upregulate cell carbohydrate metabolism through glycolysis by IPA analysis (Fig. 3). The gluconeogenesis pathway regulates production of glucose, and the glycolysis pathway leads to the breakdown of glucose for energy generation. Known as the Warburg effect, cancer cells favor glycolysis, which produces less ATP than oxidative phosphorylation, in the metabolism of glucose ${ }^{43}$. Glycolysis in GBM is upregulated ( $\sim 3$ times higher than the normal brain $)^{44}$ and promotes cell survival by suppressing apoptosis ${ }^{45}$. Glycolysis may be even more favored under hypoxia, as shown in GBM stem-like cells ${ }^{46}$. The upregulation of glycolysis suggests enhanced energy generation in GBM cells treated with CSS at $23 \mathrm{~Pa}$.

IPA analysis also identified HIFA and ARNT, encoding the two subunits HIF- $1 \alpha$ and HIF- $1 \beta$ of HIF- 1 , as the top upstream regulators activated by low level of CSS. HIF-1 is commonly overexpressed in human cancers and is associated with cell apoptosis, proliferation, invasion, and angiogenesis ${ }^{24,25}$. Although the production of HIF-1 is most commonly induced by hypoxia, growth factors and hormones can also induce HIF-1 in a normal oxygen environment ${ }^{47}$. Moreover, emerging evidence suggests that mechanical forces can trigger HIF-1 induction. For example, HIF-1 $\alpha$ can be induced by mechanical stretching in capillary endothelial cells ${ }^{48}$, by compression in skin cells $^{49}$, and by pressure in heart ventricles ${ }^{50}$. HIF-1 $\alpha$ is also upregulated in the brain cortex following traumatic brain injury ${ }^{51}$. Our results suggest that HIF-1 most likely plays a role in cell response to CSS.

To provide context with known signaling pathways, we cross-referenced differentially regulated miRNAs with known targets and multiple databases to validate and predict phenotypic behaviors (Figs. 4, 5). Use of multiple databases and hand-tagged pathway groups (Supplementary Table 3) enabled predictions and correlations independent of a single database or pathway. For each pathway, we analyzed percent overlap, which indicates the percent of target mRNAs that were differentially regulated in a given pathway, as well as the cumulative absolute 
FC, which is the summed magnitude of signaling change for all mRNAs in a given pathway. Percent overlap is a normalized measured of how many target mRNA in a given pathway were differentially regulated. Conversely, cumulative absolute FC is a measure of how significantly each pathway was differentially regulated independent of numbers of target mRNAs effected.

Unsurprisingly, the Cancer pathway group, containing classical pathways related to cell signaling in cancer, was the most influenced pathway group by percent overlap (Figs. 4B, 5A) (i.e., numbers of mRNA). We used the Cancer pathway group as a relative marker to understand the significance of changes in other pathways relevant to GBMs. For example, the KEGG Glioma pathway has several signaling outcomes, including migration, proliferation, and survival. The inclusion of the additional pathway groups helps predict which outcomes are likely to occur in this model. The Motility and Cell Cycle Pathway groups were the most strongly influenced (Fig. 5A,B). Given their low \%overlap, this suggests that, for these pathways, downstream transduction is implemented by larger changes in expression of a few RNAs as opposed to small changes in many RNAs. Both of these pathways were also highlighted by IPA (Fig. 3), validating experimental observations and providing strong prediction of cell cycle regulation changes in response to CSS. IPA also suggested a decrease in proliferation pathways (Fig. 3). GBM cells are typically dichotomous, exhibiting either migratory or proliferatory phenotypes ${ }^{52}$. Apoptosis is often suppressed in migrating GBM cell ${ }^{53,54}$, making them less susceptible to anti-proliferative therapies. However, we observed increased migration with predicted decreases in proliferation without significant changes in the Apoptosis pathway group (Fig. 5A,B). This may simply be a result of the short duration of this study (i.e., $18 \mathrm{hr}$ exposure to CSS) or may indicate more complex signaling. Increased migration into the brain parenchyma enables cells to escape focal treatments (i.e., surgical resection, radiotherapy), whereas decreased proliferation correlates with suppressed apoptosis. Thus, these findings are consistent with features of tumor aggressiveness. The heterogeneous cellular response to CSS, with LN229 displaying increased migration at low CSS and decreases at high CSS, whereas U251 cells were relatively insensitive to compression, indicates potential for both patient-to-patient variability in CSS response as well as variability within individual tumors. This variation likely depends on the genetic signature of each cell type, but indicates the potential for formation of an aggressive, migratory phenotype with poor therapeutic response to GBM drugs primarily targeting proliferating cells.

Interaction analysis identified some miRNA signaling molecules important in the CSS response. The miR548 family consisting of mir-548aj, mir-548az, and mir-548t demonstrated the largest influence, despite relatively low FC (i.e., $-2.16,-2.03$, and -2.3 , respectively) (Fig. 5E,F). These FCs were in the bottom half of absolute FC among all 27 differentially regulated precursor miRNA (Table 3). The high cumulative absolute FC of the mir548 family suggests that regulation likely occurs via large changes in a small number of target mRNA. Compared to normal brain tissue, the miR548 family is typically downregulated in GBM, suggesting a protective, prognostic role in the healthy brain ${ }^{32}$. Expression also correlates with survival, with mir $548 \mathrm{~d}-3 \mathrm{p} /-5 \mathrm{p}$ upregulated in long-term survival patients ${ }^{33}$. This correlation is a critical finding as, to the best of our knowledge, there are currently no recognized miRNAs linked to GBM mechanobiology. Thus, these data provide an important first step toward mining the mechanical tumor microenvironment for potential drug targets. Further studies in models of increased microenvironment complexity (e.g., stromal, immune cells, extracellular matrix components, 3D culture), that investigate downstream interactors, and genetic confirmation of miR548 activity would strengthen these claims. With experimental evidence provided in this mechanobiology study and in gross tumor studies by others $^{32,33}$, the miR548 family may be a candidate for pharmaceutical intervention when targeting this microenvironment niche. These data thus provide a candidate group for pharmaceutical intervention (i.e., the miR548 family) identified by leveraging the transcriptome, a cornucopia of signaling literature, and miRNA-mRNA interactions.

\section{Materials and Methods}

Cell culture. Two commonly used glioblastoma cell lines, LN229 and U251 (ATCC), were employed to study the effects of compression in GBM and provide concordance with our previous results. These cell lines were cultured using standard procedures. Cells were fed 2-3 times per week with DMEM/F12 media (Sigma-Aldrich, St. Louis, MO, USA) supplemented with 10\% fetal bovine serum (Fisher Scientific, Hampton, NH, USA), $1 \%$ penicillin/streptomycin (Invitrogen, Carlsbad, CA, USA) and 1\% MycoZap (Life Technologies, Carlsbad, CA, USA). Cells were passaged at $\sim 80 \%$ confluency.

Mechanical compression model. CSS was created using a modified version of the model described in Tse et al. ${ }^{13}$ However, unlike that study in which cells were cultured in a semi-confluent and patterned monolayer, cells in our model were cultured in a non-confluent monolayer. Cells were therefore compressed in 1D, similar to radial forces experienced by GBM cells. Briefly, cells were cultured on the membrane of a Transwell@ insert $\left(\mathrm{VWR}^{\circledR}\right.$ tissue culture plate inserts, polyester membrane, $12 \mathrm{~mm}$ diameter, $0.4 \mu \mathrm{m}$ pore size) in a 12 -well tissue culture plate (VWR). Cell culture media was added to the inner well. This pore size was selected to allow nutrient and oxygen exchange and prevent transmigration across the membrane. An agar cushion was placed atop the cells to protect against edge effects ${ }^{36}$ and from contact with the rigid weight discs used to create CSS. To prepare the agar cushion, $1 \mathrm{wt} \%$ agar solution was heated at $90^{\circ} \mathrm{C}$ for $\sim 5 \mathrm{~min}$ or until translucent. This solution was then poured into a $100 \mathrm{~mm}$ cell culture dish, allowed to set for $\sim 5-10 \mathrm{~min}$, and sterilized under ultra-violet (UV) light for 20 minutes. The secured agar gel was punched out to a size $\sim 3.5 \mathrm{~mm}$ thick and $10 \mathrm{~mm}$ in diameter with an arch punch (Grainger, Columbus, OH, USA). Finally, varying numbers of weight discs made of stainless steel, aluminum, or vinyl were placed on top of the agar cushion to apply a known compression level (Supplementary Fig. 1A). Specifically, compressions of $13,23,47$, and 115 Pa were employed, with an error of approximately \pm $0.5 \mathrm{~Pa}$. Control conditions included samples with no weight or with agar cushions only. To prevent disc oxidation or other disc-related changes in cell phenotype, discs were coated with Silastic (Dow Corning). Weight discs were sterilized in ethanol under UV for $40 \mathrm{~min}$ and dried completely prior to use. 
Wound healing assay. Collective migration was assessed using the wound healing gap assay. An Ibidi ${ }^{\circledR} 2$ well chamber-insert (Ibidi 81176) was used to pattern a consistent $500 \mu \mathrm{m}$-wide cell-free gap on the Transwell $\odot$ membrane. Cells were stained with CellTracker Green; $2 \times 10^{5}$ cells were seeded in $70 \mu \mathrm{L}$ full media in each side of the Ibidi chamber-insert and allowed to adhere for $6 \mathrm{hr}$. Then, $1 \mathrm{~mL}$ of media was added to the outer well of the Transwell@ insert, and the Ibidi chamber was removed. Agar cushions and weight discs were then added to create the desired mechanical compression. One image per insert was taken at $4 \mathrm{x}$ magnification in the same location at $0 \mathrm{hr}$ and $18 \mathrm{hr}$ after culture using an Olympus IX 71 inverted fluorescence microscope with a FITC filter. The cell-free area was quantified at the initial and final time points using ImageJ (Supplementary Fig. 1B,C), and wound closure calculated according to Eq. 1. To account for culture variations, differential wound closure is reported, which is the average difference in wound closure between each compression condition and the control (Eq. 2).

$$
\begin{aligned}
\text { Wound Closure }(W C) & =\frac{\text { Area }_{\text {initial }}-\text { Area }_{\text {final }}}{\text { Area }_{\text {initial }}} \\
\Delta \text { Wound Closure } & =\overline{W C_{\text {exp }}-W C_{c t r l}}
\end{aligned}
$$

Morphological analysis. Cells were stained with CellTracker Green (CMFDA, Invitrogen), seeded at $1 \times 10^{5}$ cells per Transwell $\odot$ insert $(\mathrm{N}=3$ per condition) in a 12 well plate, and allowed to adhere for $24 \mathrm{hr}$. Then, an agar cushion was applied (for agar control and compression conditions), and weight discs were added (for compression conditions) to obtain the desired compression. After $18 \mathrm{hr}$ of applied CSS, cells were imaged at 20x in 3 random locations per insert using an inverted fluorescence microscope with a FITC filter (Olympus IX 71). Cell morphology was characterized using Image J to determine the aspect ratio (AR) and area of cells in each images. Incomplete cells (on the edge) or dividing cells were excluded.

$\mathrm{AR}$ and cell area histograms were plotted in MATLAB to detect distribution patterns. AR distribution was plotted and fit to 1-term or 2-term probability density function (PDF) using the curve fitting tool in MATLAB with the following equations:

$$
\begin{gathered}
x=A R-1 \\
1-\text { term PDF: } f(x)=a 1 \times \frac{1}{x} \times \frac{1}{\sigma 1 \sqrt{2 \pi}} \exp \left(-\frac{(\ln x-\mu 1)^{2}}{2 \sigma 1^{2}}\right) \\
2-\operatorname{term~PDF:~} f(x)=a 1 \times \frac{1}{x} \times \frac{1}{\sigma 1 \sqrt{2 \pi}} \exp \left(-\frac{(\ln x-\mu 1)^{2}}{2 \sigma 1^{2}}\right)+a 2 \times \frac{1}{x} \times \frac{1}{\sigma 2 \sqrt{2 \pi}} \exp \left(-\frac{(\ln x-\mu 2)^{2}}{2 \sigma 2^{2}}\right)
\end{gathered}
$$

$\mathrm{X}$ in Eq. 1 was set to be AR-1 because the value of AR is at least 1 . Scaling parameters, a1 and a2, determine the percentage of each fitted equation. The distribution curve means and standard deviations are given by $\mu 1, \mu 2$ and $\sigma 1, \sigma 2$, respectively. The mode $(\mathrm{m})$, which represents the peak location of each curve, is determined by:

$$
\mathrm{m}=\exp \left(\mu-\sigma^{2}\right)+1
$$

Goodness of fit was compared by the value of sum of squares for error (SSE) and $\mathrm{R}^{2}$ reported by MATLAB. The fitting that resulted in the smaller SSE and higher $\mathrm{R}^{2}$ was presented.

RNA extraction. LN229 cells treated with no compression or compression at $23 \mathrm{~Pa}$ for $18 \mathrm{hr}$ were processed for microarray analysis. Briefly, LN229 cells were patterned using the Ibidi chambers and cultured in a Transwell(C) insert without staining as described above. To ensure even compression over the whole cell population, one side of the Ibidi chamber was cut off, placed in the center of the Transwell@ membrane, and used for cell patterning. No compression was added to the control. After $18 \mathrm{hr}$ of incubation, Transwell@ inserts were washed 3x with PBS and immersed in TRIzol reagent (ThermoFisher 15596026) for RNA extraction following the manufacturer's instructions. Extracted RNA was further purified with an RNA clean-up and concentration kit (Norgen 23600) and tested for quality by Nanodrop analysis and a TapeStation RNA Bioanalyzer. RNA samples with a $260 \mathrm{~nm} / 230 \mathrm{~nm}$ absorbance ratio $>1$, a $260 \mathrm{~nm} / 280 \mathrm{~nm}$ absorbance ratio $>1.9$, and a RNA integrity number $(\mathrm{RIN})>7$ were used for microarray analysis as recommended by the manufacturer. Experimental replicates $(\mathrm{N}=4$ for each group) were prepared in individual inserts and treated with $23 \mathrm{~Pa}$ of compression on different days.

Microarray and pathway analysis. Microarray analysis was performed using the GeneChip ${ }^{\mathrm{TM}}$ Human Transcriptome Array 2.0 (Applied Biosystems ${ }^{\mathrm{TM}}$ ) following manufacturer's instructions. Briefly, $100 \mathrm{ng}$ RNA was used to generate ss-cDNA according to the manufacturer's protocol in the GeneChip ${ }^{\circledR}$ WT PLUS Reagent Kit, and $15 \mu \mathrm{g}$ of labeled, fragmented, and biotin-labeled ss-cDNA was hybridized at $45^{\circ} \mathrm{C}$ for $16 \mathrm{hr}$ at $60 \mathrm{rpm}$ in an Affymetrix 645 Hybridization oven. GeneChips were then washed and stained using an Affymetrix 450 Fluidics Station, and scanned with an Affymetrix 30007 G GeneChip Scanner. Data files were generated and processed with an Affymetrix software and Expression Console. The RNA quality test and microarray were performed by the genomics core at The Ohio State University. Gene expression analysis was performed using the Ebayes ANOVA method in the Transcriptome Analysis Console (TAC 4.01, ThermoFisher) with false discovery rate 
(FDR) adjusted $\mathrm{p}<0.05$. Heat maps of gene expression were generated with hierarchical clustering. A total of 489 differential expressed genes $(\mathrm{FDR}<0.05, \mathrm{FC}>2$ ) were uploaded into the Ingenuity Pathway Analysis server (http://www.ingenuity.com) to identify pathways/disease/functions regulated by low CSS.

miR-mRNA interaction analysis. For each precursor miRNA differentially regulated in the microarray, an Excel file was downloaded from miRPathDB that included statistically significant, over-represented pathways and target mRNA in the pathway. If unavailable, miRNA were substituted for a similar sequence or identical sequence. For example, hsa-mir-548-aj was substituted for mir-548-aj2. The ' 2 ' in mir-548-aj2 indicates different precursor miRNA that yield identical sequences. Both $5 \mathrm{p} / 3 \mathrm{p}$ isomiRs were included to match HTA 2.0 coverage. Then, pathways of interest were hand-tagged into pathway groups classified as Cancer, Motility, DNA Repair, Cell Cycle, Apoptosis, Miscellaneous, or Canonical (Supplementary Table 3) using explicit inclusion/exclusion criteria to avoid bias (Supplementary Table 4). Using Python scripts (Supplementary File 1-8), each miRNA excel file was first cross-referenced with the list of differentially regulated mRNA from the microarray to annotate the file with targets in each pathway that were differentially regulated. Then, the pathway groups were consolidated into a single list with duplicates removed. Finally, heat maps were generated for the number of intersections, percent overlap, and cumulative absolute FC. For number of intersections, each node is the number of intersections for each pathway. For percent overlap and cumulative FC, each node of the corresponding heat map is the intersection of a miRNA and a single pathway. Percent overlap is the number of differentially regulated target mRNA in the pathway normalized to the number of total target mRNA in the pathway. Cumulative absolute FC is the sum of the absolute FC for each differentially regulated target mRNA in the pathway. Individual node values were used for averages for mir groups, pathway groups, and miRNA.

Statistical analysis. All data were presented as mean with standard error. Wound healing experiments were conducted as matched pair experiments between the experimental condition and the control for each replicate. This allowed us to account for day-to-day variability. Post-hoc one-way t-test $\alpha$ levels were adjusted with Bonferroni corrections to 0.01 for 5 comparisons. The remainder of the data was compared using ANOVA and the Tukey-Kramer HSD method. These analyses were performed with a significance level of $\alpha=0.05$. All data was analyzed in JMP Pro 14 statistical software.

Received: 10 September 2019; Accepted: 16 December 2019;

Published online: 15 January 2020

\section{References}

1. Stupp, R. et al. Effect of Tumor-Treating Fields Plus Maintenance Temozolomide vs Maintenance Temozolomide Alone on Survival in Patients With Glioblastoma A Randomized Clinical Trial. Jama-J Am Med Assoc 318, 2306-2316, https://doi.org/10.1001/ jama.2017.18718 (2017).

2. Cuddapah, V. A., Robel, S., Watkins, S. \& Sontheimer, H. A neurocentric perspective on glioma invasion. Nat. Rev. Neurosci. 15, 455-465, https://doi.org/10.1038/nrn3765 (2014).

3. Giese, A. et al. Dichotomy of astrocytoma migration and proliferation. International journal of cancer 67, 275-282, 10.1002/ (SICI) 1097-0215(19960717)67:2<275::AID-IJC20>3.0.CO;2-9 (1996).

4. Chaichana, K. L. et al. Establishing percent resection and residual volume thresholds affecting survival and recurrence for patients with newly diagnosed intracranial glioblastoma. Neuro Oncol 16, 113-122, https://doi.org/10.1093/neuonc/not137 (2014).

5. Hochberg, F. H. \& Pruitt, A. Assumptions in the radiotherapy of glioblastoma. Neurology 30, 907-907, https://doi.org/10.1212/ wnl.30.9.907 (1980).

6. Hirata, E. et al. In vivo fluorescence resonance energy transfer imaging reveals differential activation of Rho-family GTPases in glioblastoma cell invasion. J. Cell Sci. 125, 858-868, https://doi.org/10.1242/jcs.089995 (2012).

7. Kane, J. R. The Role of Brain Vasculature in Glioblastoma. Mol. Neurobiol., https://doi.org/10.1007/s12035-019-1561-y (2019).

8. Watkins, S. et al. Disruption of astrocyte-vascular coupling and the blood-brain barrier by invading glioma cells. Nat. Commun. $\mathbf{5}$, 4196, https://doi.org/10.1038/ncomms5196 (2014).

9. Padera, T. P. et al. Pathology: cancer cells compress intratumour vessels. Nature 427, 695, https://doi.org/10.1038/427695a (2004).

10. Boucher, Y. \& Jain, R. K. Microvascular pressure is the principal driving force for interstitial hypertension in solid tumors: implications for vascular collapse. Cancer Res. 52, 5110-5114 (1992).

11. Jain, R. K., Martin, J. D. \& Stylianopoulos, T. The Role of Mechanical Forces in Tumor Growth and Therapy. Annu. Rev. Biomed. Eng. 16, 321-346, https://doi.org/10.1146/annurev-bioeng-071813-105259 (2014).

12. Seano, G. et al. Solid stress in brain tumours causes neuronal loss and neurological dysfunction and can be reversed by lithium. Nature Biomedical Engineering 3, 230-+, https://doi.org/10.1038/s41551-018-0334-7 (2019).

13. Tse, J. M. et al. Mechanical compression drives cancer cells toward invasive phenotype. PNAS 109, 911-916, https://doi.org/10.1073/ pnas.1118910109 (2012).

14. Demou, Z. N. Gene Expression Profiles in 3D Tumor Analogs Indicate Compressive Strain Differentially Enhances Metastatic Potential. Ann Biomed Eng 38, 3509-3520, https://doi.org/10.1007/s10439-010-0097-0 (2010).

15. Cheng, G., Tse, J., Jain, R. K. \& Munn, L. L. Micro-Environmental Mechanical Stress Controls Tumor Spheroid Size and Morphology by Suppressing Proliferation and Inducing Apoptosis in Cancer Cells. PLOS ONE 4, e4632, https://doi.org/10.1371/journal. pone.0004632 (2009).

16. Helmlinger, G., Netti, P. A., Lichtenbeld, H. C., Melder, R. J. \& Jain, R. K. Solid stress inhibits the growth of multicellular tumor spheroids. Nature biotechnology 15, 778-783, https://doi.org/10.1038/nbt0897-778 (1997).

17. Mammoto, T. et al. Role of collagen matrix in tumor angiogenesis and glioblastoma multiforme progression. The American journal of pathology 183, 1293-1305, https://doi.org/10.1016/j.ajpath.2013.06.026 (2013).

18. Nia, H. T. et al. Solid stress and elastic energy as measures of tumour mechanopathology. Nat Biomed Eng 1, https://doi.org/10.1038/ s41551-016-0004 (2016).

19. Winkler, F. et al. Imaging glioma cell invasion in vivo reveals mechanisms of dissemination and peritumoral angiogenesis. Glia 57, 1306-1315, https://doi.org/10.1002/glia.20850 (2009).

20. Gritsenko, P., Leenders, W. \& and cell biology, F.-P. Recapitulating in vivo-like plasticity of glioma cell invasion along blood vessels and in astrocyte-rich stroma. Histochem. Cell Biol. (2017).

21. Parsons, J. T., Horwitz, A. R. \& Schwartz, M. A. Cell adhesion: integrating cytoskeletal dynamics and cellular tension. Nat. Rev. Mol. Cell Biol. 11, 633-643, https://doi.org/10.1038/nrm2957 (2010). 
22. Tian, Y. et al. MicroRNAs let-7b/i suppress human glioma cell invasion and migration by targeting IKBKE directly. Biochem. Biophys. Res. Commun. 458, 307-312, https://doi.org/10.1016/j.bbrc.2015.01.105 (2015).

23. Lee, S. T. et al. Let-7 microRNA inhibits the proliferation of human glioblastoma cells. J. Neurooncol. 102, 19-24, https://doi. org/10.1007/s11060-010-0286-6 (2011).

24. Semenza, G. L. Targeting HIF-1 for cancer therapy. Nat Rev Cancer 3, 721-732, https://doi.org/10.1038/nrc1187 (2003).

25. Carmeliet, P. et al. Role of HIF-1 alpha or in hypoxia-mediated apoptosis, cell proliferation and tumour angiogenesis. Nature 394, 485-490, https://doi.org/10.1038/28867 (1998).

26. Cano, C. E., Hamidi, T., Sandi, M. J. \& Iovanna, J. L. Nupr1: The Swiss-Knife of Cancer. Journal Of Cellular Physiology 226, 1439-1443, https://doi.org/10.1002/jcp.22324 (2011).

27. Chowdhury, U., Samant, R., Fodstad, O. \& Shevde, L. Emerging role of nuclear protein 1 (NUPR1) in cancer biology. Cancer Metast Rev 28, 225-232, https://doi.org/10.1007/s10555-009-9183-x (2009).

28. Backes, C. et al. miRPathDB: a new dictionary on microRNAs and target pathways. Nucleic Acids Res. 45, D90-D96, https://doi. org/10.1093/nar/gkw926 (2016).

29. Hegi, M. E. et al. Clinical Trial Substantiates the Predictive Value of $<$ strong $><$ em $>$ O-6-Methylguanine-DNA Methyltransferase $<$ / $\mathrm{em}></$ strong $>$ Promoter Methylation in Glioblastoma Patients Treated with Temozolomide. Clin. Cancer Res. 10, 1871-1874, https://doi.org/10.1158/1078-0432.Ccr-03-0384 (2004).

30. Friedman, H. S. et al. DNA mismatch repair and O6-alkylguanine-DNA alkyltransferase analysis and response to Temodal in newly diagnosed malignant glioma. J. Clin. Oncol. 16, 3851-3857, https://doi.org/10.1200/jco.1998.16.12.3851 (1998).

31. Yang, L. et al. Role of the microRNA 181 family in glioma development. Molecular medicine reports 17, 322-329, https://doi. org/10.3892/mmr.2017.7895 (2018)

32. Hermansen, S. K. et al. A 4-miRNA signature to predict survival in glioblastomas. PLoS One 12, e0188090, https://doi.org/10.1371/ journal.pone.0188090 (2017).

33. Herman, A. et al. Analysis of Glioblastoma Patients' Plasma Revealed the Presence of MicroRNAs with a Prognostic Impact on Survival and Those of Viral Origin. PLoS One 10, e0125791, https://doi.org/10.1371/journal.pone.0125791 (2015).

34. Liu, B., Shyr, Y., Cai, J. \& Liu, Q. Interplay between miRNAs and host genes and their role in cancer. Briefings in Functional Genomics 18, 255-266, https://doi.org/10.1093/bfgp/elz002 (2019).

35. Calhoun, M. A., Chowdhury, S. S., Nelson, M. T. \& Nanomaterials, L.-J. J. Effect of Electrospun Fiber Mat Thickness and Support Method on Cell Morphology. Nanomaterials-Basel (2019).

36. Rao, S. S. et al. Inherent Interfacial Mechanical Gradients in 3D Hydrogels Influence Tumor Cell Behaviors. PLoS One 7, e35852, https://doi.org/10.1371/journal.pone.0035852 (2012).

37. Qazi, H., Shi, Z. D. \& one, T.-J. M. Fluid shear stress regulates the invasive potential of glioma cells via modulation of migratory activity and matrix metalloproteinase expression. PLoS One (2011).

38. Alenghat, F. J. \& Ingber, D. E. Mechanotransduction: All Signals Point to Cytoskeleton, Matrix, and Integrins. Sci. STKE 2002, pe6-pe6, https://doi.org/10.1126/stke.2002.119.pe6 (2002).

39. Ata, R. \& Antonescu, C. N. Integrins and Cell Metabolism: An Intimate Relationship Impacting Cancer. Int. J. Mol. Sci. 18, 189 (2017).

40. Yang, L. et al. Twist promotes reprogramming of glucose metabolism in breast cancer cells through PI3K/AKT and p53 signaling pathways. Oncotarget 6, 25755-25769, https://doi.org/10.18632/oncotarget.4697 (2015).

41. Haskin, C., Cameron, I. \& Athanasiou, K. Physiological Levels of Hydrostatic-Pressure Alter Morphology and Organization of Cytoskeletal and Adhesion Proteins in Mg-63 Osteosarcoma Cells (Vol 71, Pg 27, 1993). Biochem Cell Biol 71, 313-313, https://doi. org/10.1139/093-047 (1993).

42. Scherer, H. J. Structural Development in Gliomas. Cancer Res. 34(0400).

43. Warburg, O. Origin of Cancer Cells. Science 123, 309-314, https://doi.org/10.1126/science.123.3191.309 (1956).

44. Oudard, S. et al. High glycolysis in gliomas despite low hexokinase transcription and activity correlated to chromosome 10 loss. $\mathrm{Br}$. J. Cancer 74, 839-845, https://doi.org/10.1038/bjc.1996.446 (1996).

45. Wolf, A., Agnihotri, S. \& Guha, A. Targeting Metabolic Remodeling in Glioblastoma Multiforme. Oncotarget 1, 552-562 (2010).

46. Kathagen, A. et al. Hypoxia and oxygenation induce a metabolic switch between pentose phosphate pathway and glycolysis in glioma stem-like cells. Acta Neuropathologica 126, 763-780, https://doi.org/10.1007/s00401-013-1173-y (2013).

47. Richard, D. E., Berra, E. \& Pouyssegur, J. Nonhypoxic pathway mediates the induction of hypoxia-inducible factor 1 alpha in vascular smooth muscle cells. J. Biol. Chem. 275, 26765-26771 (2000).

48. Milkiewicz, M. \& Haas, T. L. Effect of mechanical stretch on HIF-1 alpha and MMP-2 expression in capillaries isolated from overloaded skeletal muscles: laser capture microdissection study. Am J Physiol-Heart C 289, H1315-H1320, https://doi.org/10.1152/ ajpheart.00284.2005 (2005).

49. Kaneko, M. et al. Compression-induced HIF-1 enhances thrombosis and PAI-1 expression in mouse skin. Wound Repair Regen 23, 657-663, https://doi.org/10.1111/wrr.12312 (2015).

50. Shyu, K. G., Liou, J. Y., Wang, B. W., Fang, W. J. \& Chang, H. Carvedilol prevents cardiac hypertrophy and overexpression of hypoxiainducible factor-1 alpha and vascular endothelial growth factor in pressure-overloaded rat heart. J Biomed Sci 12, 409-420, https:// doi.org/10.1007/s11373-005-3008-x (2005).

51. Li, A. H., Sun, X. L., Ni, Y. H., Chen, X. \& Guo, A. S. HIF-1 alpha Involves in Neuronal Apoptosis after Traumatic Brain Injury in Adult Rats. J Mol Neurosci 51, 1052-1062, https://doi.org/10.1007/s12031-013-0084-7 (2013).

52. Giese, A., Bjerkvig, R., Berens, M. E. \& Westphal, M. Cost of migration: invasion of malignant gliomas and implications for treatment. Journal of clinical oncology: official journal of the American Society of Clinical Oncology 21, 1624-1636, https://doi. org/10.1200/JCO.2003.05.063 (2003).

53. Mariani, L. et al. Glioma Cell Motility is Associated with Reduced Transcription of Proapoptotic and Proliferation Genes: A cDNA Microarray Analysis. Journal of neuro-oncology 53, 161-176, https://doi.org/10.1023/a:1012253317934 (2001).

54. Cho, S. Y. \& Klemke, R. L. Extracellular-Regulated Kinase Activation and Cas/Crk Coupling Regulate Cell Migration and Suppress Apoptosis during Invasion of the Extracellular Matrix. The Journal of Cell Biology 149, 223-236, https://doi.org/10.1083/ jcb.149.1.223 (2000).

55. Su, J. Y. et al. Stanniocalcin-1, a new biomarker of glioma progression, is associated with prognosis of patients. Tumor Biology 36, 6333-6339, https://doi.org/10.1007/s13277-015-3319-0 (2015).

56. Schmit, K. \& Michiels, C. TMEM Proteins in Cancer: A Review. Frontiers in Pharmacology 9, doi:ARTN 1345 https://doi. org/10.3389/fphar.2018.01345 (2018).

57. Shin, H. J. et al. Carbonic anhydrase IX (CA9) modulates tumor-associated cell migration and invasion. Journal of Cell Science 124, 1077-1087, https://doi.org/10.1242/jcs.072207 (2011).

58. Nakada, M. et al. Integrin alpha3 is overexpressed in glioma stem-like cells and promotes invasion. Br. J. Cancer 108, 2516-2524, https://doi.org/10.1038/bjc.2013.218 (2013).

59. Robertson, E., Perry, C., Doherty, R. \& Madhusudan, S. Transcriptomic profiling of Forkhead box transcription factors in adult glioblastoma multiforme. Cancer Genomics Proteomics 12, 103-112 (2015).

60. Liang, Y. et al. Gene expression profiling reveals molecularly and clinically distinct subtypes of glioblastoma multiforme. Proc. Natl. Acad. Sci. USA 102, 5814-5819, https://doi.org/10.1073/pnas.0402870102 (2005). 
61. Freije, W. A. et al. Gene expression profiling of gliomas strongly predicts survival. Cancer Res. 64, 6503-6510, https://doi. org/10.1158/0008-5472.CAN-04-0452 (2004).

62. Romao, L. F. et al. Connective tissue growth factor (CTGF/CCN2) is negatively regulated during neuron-glioblastoma interaction. PLoS One 8, e55605, https://doi.org/10.1371/journal.pone.0055605 (2013).

63. Treps, L., Perret, R., Edmond, S., Ricard, D. \& Gavard, J. Glioblastoma stem-like cells secrete the pro-angiogenic VEGF-A factor in extracellular vesicles. J Extracell Vesicles 6, 1359479-1359479, https://doi.org/10.1080/20013078.2017.1359479 (2017).

64. Yang, X. et al. VEGF-B promotes cancer metastasis through a VEGF-A-independent mechanism and serves as a marker of poor prognosis for cancer patients. Proceedings of the National Academy of Sciences 112, E2900-E2909, https://doi.org/10.1073/ pnas.1503500112 (2015).

65. Srinivasan, S., Patric, I. R. \& Somasundaram, K. A ten-microRNA expression signature predicts survival in glioblastoma. PLoS One 6, e17438, https://doi.org/10.1371/journal.pone.0017438 (2011).

66. Ng, W. L., Yan, D., Zhang, X., Mo, Y. Y. \& Wang, Y. Over-expression of miR-100 is responsible for the low-expression of ATM in the human glioma cell line: M059J. DNA Repair (Amst) 9, 1170-1175, https://doi.org/10.1016/j.dnarep.2010.08.007 (2010).

67. Moller, H. G. et al. A systematic review of microRNA in glioblastoma multiforme: micro-modulators in the mesenchymal mode of migration and invasion. Mol. Neurobiol. 47, 131-144, https://doi.org/10.1007/s12035-012-8349-7 (2013).

68. Huang, S. X. et al. Upregulation of miR-181a suppresses the formation of glioblastoma stem cells by targeting the Notch2 oncogene and correlates with good prognosis in patients with glioblastoma multiforme. Biochem. Biophys. Res. Commun. 486, 1129-1136, https://doi.org/10.1016/j.bbrc.2017.04.008 (2017).

69. Liu, L., Cui, S., Zhang, R., Shi, Y. \& Luo, L. MiR-421 inhibits the malignant phenotype in glioma by directly targeting MEF2D. Am J Cancer Res 7, 857-868 (2017).

70. Li, S. et al. miR-423-5p contributes to a malignant phenotype and temozolomide chemoresistance in glioblastomas. Neuro Oncol 19, 55-65, https://doi.org/10.1093/neuonc/now129 (2017).

71. Shao, N. et al. miR-454-3p Is an Exosomal Biomarker and Functions as a Tumor Suppressor in Glioma. Mol. Cancer Ther. 18, 459-469, https://doi.org/10.1158/1535-7163.MCT-18-0725 (2019).

\section{Acknowledgements}

The authors acknowledge support of the Pelotonia Cancer Research Fellowship (MAC). These are the words and opinions of the authors only and do not reflect those of the Pelotonia program. This work was supported by the National Science Foundation [DGE0221678, CMMI0747252] and the National Institutes of Health R01HL132355 and UL1TR002733 (Center for Clinical and Translational Sciences).

\section{Author contributions}

M.A.C., Y.C., J.J.O. and J.O.W. designed the experiments. M.A.C., Y.C. and E.EE. conducted migration and morphology experiments; M.A.C., Y.C., E.E.E. and J.O.W. interpreted and analyzed this data. Y.C. conducted the microarray experiment; Y.C., X.M. and J.J.O. analyzed microarray data. Y.C. and X.M. conducted I.P.A. analysis. M.A.C. conducted miRNA-MRNA interaction analysis. All authors wrote and edited the paper.

\section{Competing interests}

The authors declare no competing interests.

\section{Additional information}

Supplementary information is available for this paper at https://doi.org/10.1038/s41598-019-56983-X.

Correspondence and requests for materials should be addressed to J.O.W.

Reprints and permissions information is available at www.nature.com/reprints.

Publisher's note Springer Nature remains neutral with regard to jurisdictional claims in published maps and institutional affiliations.

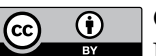

Open Access This article is licensed under a Creative Commons Attribution 4.0 International

License, which permits use, sharing, adaptation, distribution and reproduction in any medium or format, as long as you give appropriate credit to the original author(s) and the source, provide a link to the Creative Commons license, and indicate if changes were made. The images or other third party material in this article are included in the article's Creative Commons license, unless indicated otherwise in a credit line to the material. If material is not included in the article's Creative Commons license and your intended use is not permitted by statutory regulation or exceeds the permitted use, you will need to obtain permission directly from the copyright holder. To view a copy of this license, visit http://creativecommons.org/licenses/by/4.0/.

(c) The Author(s) 2020 